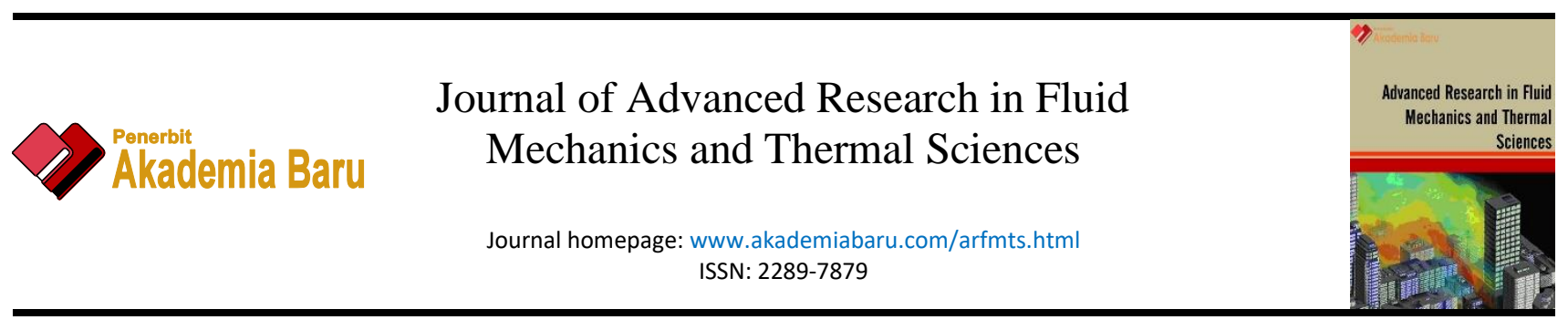

\title{
Two-Phase Region Effect on Film Condensation on an Inclined Plate Embedded in a Porous Medium
}

\author{
Yee Lee Yeu ${ }^{1, *}$, Alexander Gorin ${ }^{1}$ \\ 1 Faculty of Engineering, Computing and Science, Swinburne University of Technology (Sarawak Campus), Kuching, Sarawak, Malaysia
}

\begin{tabular}{|c|c|}
\hline ARTICLE INFO & ABSTRACT \\
\hline $\begin{array}{l}\text { Article history: } \\
\text { Received } 26 \text { June } 2020 \\
\text { Received in revised form } 19 \text { July } 2020 \\
\text { Accepted } 20 \text { July } 2020 \\
\text { Available online } 9 \text { December } 2020\end{array}$ & $\begin{array}{l}\text { Film condensation in a porous medium has been receiving increasing attention due to } \\
\text { its wide range of heat transfer applications. Some examples of these practical } \\
\text { applications are distillation, drying technology, geothermal energy, cooling towers, } \\
\text { heat exchangers, and air conditioning. One of the characteristic features of film } \\
\text { condensation in porous media is the formation of a two-phase zone separating the } \\
\text { liquid film and the vapour zone due to capillary pressure. In this paper, a physico- } \\
\text { mathematical model of liquid film condensation on a surface embedded in a porous } \\
\text { medium with a two-phase region effect is developed and presented. The model is } \\
\text { based on momentum and continuity equations as applied to the liquid film and the } \\
\text { two-phase flow region supplemented with the Darcy flow assumption and } \\
\text { assumptions on the Leverette J-function and the saturation behaviour near the edge } \\
\text { of the liquid film. The developed model allows a simple analytical solution to the } \\
\text { problem in distinction to semi-analytical and numerical solutions published by } \\
\text { different authors. From the model developed, it shows that the presence of the two- } \\
\text { phase region decreases the liquid film thickness. By taking the capillary effects into } \\
\text { consideration results in higher heat transfer and condensation rates due to the } \\
\text { decrease in the liquid film thickness. The presented model yields good agreement } \\
\text { when compared to the theoretical results and experimental data by other authors. The } \\
\text { developed model addresses the fundamental concepts of phase transition in porous } \\
\text { media which can effectively find applications in many areas. }\end{array}$ \\
\hline
\end{tabular}

\section{Keywords:}

Film Condensation; Two-Phase Region; Porous Medium; Porous Media; Heat

\section{Introduction}

Film condensation in the absence of porous medium has been extensively studied and well understood [1-7]. Nakoryakov and Gorin [8] represented a simple model for a film condensation on an inclined plate embedded in a porous medium assuming a distinct interface, constant transport

\footnotetext{
* Corresponding author.

E-mail address: yyeu@swinburne.edu.my
}

https://doi.org/10.37934/arfmts.78.2.6784 
properties, the Darcy regime for condensate film flow, and uniform porous structure across the film, which resulted in the correlation for the average heat transfer coefficient,

$\overline{N u}=\frac{\bar{\alpha}_{L} L}{\lambda_{e}}=\left(2 A r^{*} \operatorname{Pr} \mathrm{K}_{\mathrm{u}}\right)^{1 / 2}$

where $A r^{*}$ is the modified Archimedes number, $\mathrm{K}_{\mathrm{u}}$ is the phase conversion criterion or known as Kutateladze condensation number and $\mathrm{Pr}$ is the Prandtl number.

The Nakoryakov and Gorin's [8] model showed good agreement with the Cheng's [9] model, which required numerical solution of ordinary differential equation. It agreed well with experimental data by different authors too.

It should be emphasized that thermodynamics governing film condensation in the presence of porous medium differs noticeably from that in the absence of porous media. In the past decade, there has been an increased interest in research on film condensation in porous media [8-23], due to its wide range of heat transfer applications. Some examples of these practical applications are the design of heat pipes, heat exchangers, food drying, and many other applications.

Until today, there are limited numbers of experimental studies carried out to support, refute, or validate hypothesis in physical models. Dvorovenko's [18] comprehensive experimental study of heat transfer in film condensation on a plate embedded in a packed bed is deserving of more attention. Porous media were modelled by using glass beads of various sizes placed on a plate length changing from $250 \mathrm{~mm}$ to $1000 \mathrm{~mm}$. The temperature profiles across the liquid film thickness of condensate of refrigerant- 12 were measured by seven precise thermocouples placed across the porous layer. The heat flux was also measured and recorded. The results could be considered of the highest quality of data possible.

Despite the long list of the study of condensation in porous media, the understanding of condensation in porous media with a two-phase region effect still not well understood. Two-phase zone or two-phase region is where the liquid and vapour coexist at a condition of local equilibrium. The existence of the two-phase region is due to the surface tension and capillary effect which can result in liquid being sucked into fine pores. In the two-phase region, the liquid phase and vapour phase are flowing in a counter-current direction. Capillary pressure and relative permeability are the key parameters governing the fluid flow in a porous medium. Although many researchers did not consider the surface tension effect in their theoretical investigation [9, 14-15, 24-26], many experimental studies have reported that a two-phase zone is formed when a confined watersaturated porous medium is heated [27-30].

Udell [31] conducted an experiment to study heat and mass transfer characteristics in porous media. The porous media were formed using coarse and fine sand with a grain size of 833-589 $\mu \mathrm{m}$ and 208-149 $\mu \mathrm{m}$ respectively. The porous test samples were heated the top and cooled at the bottom. From the experimental results, the author identified three distinct regions at steady-state conditions. These three distinct zones were the vapour at the upper region, liquid at the lower region, and the region separating the upper vapour zone and the lower liquid zone is a two-phase zone. The length of the two-phase zone of the fine grain sand porous sample was longer than the coarse grain sand porous sample at comparable heat fluxes.

Udell [31] also developed a theoretical model for one-dimensional, steady-state heat and mass transfer in porous media saturated with the liquid and vapour phases of a single component fluid. The model is examined with various gravitational orientations and medium permeability to predict the length of the two-phase zone as a function of the heat flux, fluid properties, and porous medium characteristic. Udell [32] then extended his study by including the effects of the capillary, gravity forces, and phase change. He found that the two-phase zone was nearly isothermal and convection 
dominated. The temperature, pressure, and saturation profiles were obtained corresponding to various gravitational orientations in the two-phase zone. The conditions in which the gravitational effects can be neglected and the criteria to determine the dry-out heat flux were identified. Apparent thermal conductivities of the two-phase zone for various gravitational orientations and permeability were evaluated as well [32].

As the effective pore radii are small in many porous media applications, many researchers have drawn attention to the capillary pressure effects in porous media. Plumb [33] examined the effect of the capillary action on film condensation in porous materials theoretically. A two-phase capillary fringe that separated the liquid film from the vapour was presented in his model. He found the capillary transport plays an important role in increasing the heat transfer and condensation rates on a vertical isothermal surface as the liquid film thickness decreases due to the capillary effects. The results for both a constant temperature capillary fringe model and a constant pressure capillary fringe model are well predicted in many practical applications. Majumdar and Tien [34] studied the effects of surface tension on film condensation in a porous medium and validated the three-zone model developed by Plumb [33]. They presented that the existence of a two-phase zone lying between the liquid and the vapour regions is required to achieve the thermodynamics of phase equilibria for film condensation in a porous medium. Three models with different assumptions were suggested by Majumdar and Tien [34]. They refined the liquid film and two-phase zone by neglecting the effect of vapour flow in isothermal two-phase zone. It is due to the no-slip condition at the wall and no-shear condition at the interface with the two-phase zone.

Chung et al., [35] then continued with the development of the three-zone model. They developed a unified model combining the three models of Majumdar and Tien [34]. In their numerical solutions to the mathematical model, they include the assumption of the existence of a two-phase region between the liquid film and the vapour phase. The effect of vapour flow was included in the twophase zone. They also investigated the problem of condensation in a porous medium near a cold vertical surface experimentally. Their numerical results were compared with experimental data and show satisfactory agreement between the numerical and experimental results.

A new formulation for one-dimensional, steady-state heat and mass transfer in a porous medium with a two-phase zone was presented by Bridge et al., [36]. The problem of steady filmwise condensation on a finite size horizontal plate embedded in a porous medium with the effect of surface tension was studied by Chang [37]. A non-dimensionless parameter $B o_{c}$ was introduced. It gives the ratio of capillary force to the gravitational force, which is the reciprocal of the Bond number, $B o$. If the $B o$ number is small, the surface tension force would be dominant and hence the two-phase zone would contribute to the total liquid flow. The porous medium with higher $B o_{c}$ has better heat transfer performance, hence induced a greater average Nusselt number and lower liquid film thickness. The dimensionless liquid film thickness can be obtained by using the Runge-Kutta shooting method.

In one of the recent papers, Jafari et al., [38] investigated the condensation heat transfer of twophase closed thermosyphons experimentally. They proposed three Nusselt correlations to predict condensation heat transfer, which are Rohsenow [39], Hashimoto and Kaminaga [40], and Jouhara and Robinson [41]. However, these correlations do not consider the two-phase region effect and do not include the Bond number in their correlation. Bond number is a dimensionless number that measures the importance of gravitational forces compared to surface tension forces. It is used to characterize the shape of bubbles or drops moving in a surrounding fluid.

Song et al., [42-44] did systematic researches on the two-phase flow condensation. They investigated the new adiabatic and condensation two-phase flow pattern maps of R14 in a horizontal tube. They proposed a new dimensionless number that takes into account the inertia force, gravity 
force, shear force, and surface tension force. This dimensionless number is to develop the new adiabatic flow pattern transition criteria [42]. Then, they did the measurement and modeling for flow condensation heat transfer performance of tetrafluoromethane (R14) based on two-phase flow patterns [43]. Lastly, they investigated the two-phase flow condensation pressure drop of R14 in a horizontal tube experimentally and did the correlation development for frictional pressure drops [44].

Although many researchers had discovered and proved that the two-phase zone exists during condensation in porous media, the problem remains complex to solve. Furthermore, it has been more than one decade with no further development in developing the Nusselt correlation for film consideration with a two-phase region effect. So, a simple solution to this complex problem is a necessity to be studied and presented. Therefore, this paper aims to develop a physico-mathematical model of liquid film condensation on a surface embedded in a porous medium with a two-phase region effect and study the steady heat transfer in a two-phase region of a water-saturated porous medium.

\section{Mathematical Formulation}

To develop the mathematical formulation for film condensation on an inclined plate embedded in a porous medium with a two-phase region effect, the $(x, y)$ coordinate system shown in Figure 1 is introduced. The liquid film of condensate adjacent to the cold plate surface. The cold plate temperature is uniform and maintained at temperature $T_{w}$. Far away from the plate is a pure vapour region. The vapour temperature is uniform and maintained at the saturation temperature, $T_{\text {sat }}$.

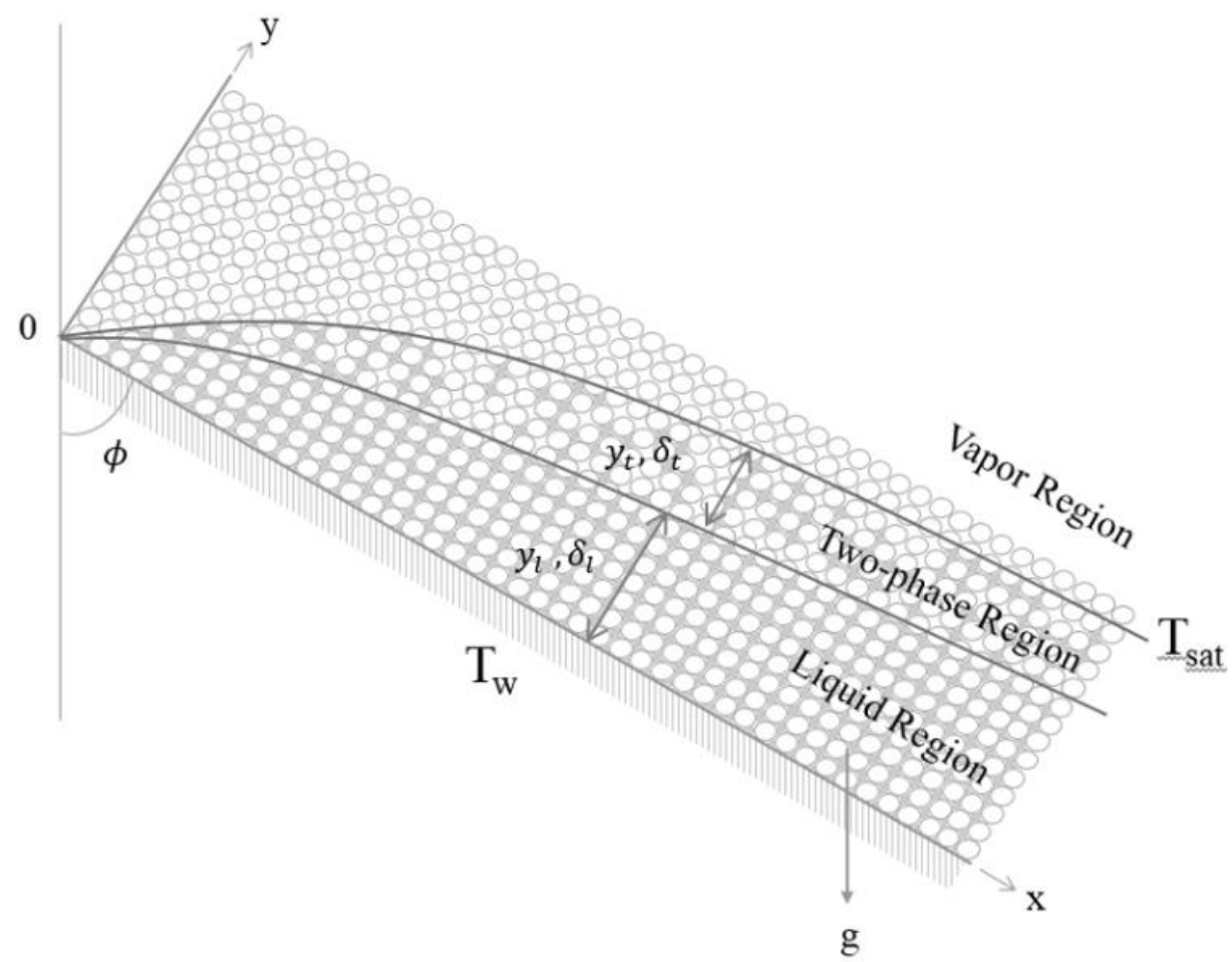

Fig. 1. Schematic diagram of film condensation with a two-phase region on an inclined plate embedded in a porous medium 
The condensation takes place on the cold plate wall embedded in a porous medium. In between the liquid and vapour regions, there is a two-phase zone where liquid and vapour co-exist at conditions of local equilibrium. The liquid film thickness $\delta_{l}$ and two-phase region film thickness $\delta_{t}$ grow continuously with increasing $x$ by reasons of liquid film flow downwards due to gravitational effect and newly condensing vapour in the downward direction.

Additional assumptions are as follows

(a) Steady-state condensation.

(b) The film thickness exceeds much the pore size, $\delta \gg d$.

(c) Darcy's flow in both liquid zone and two-phase zone.

(d) Change in kinetic energy and potential energy within the film flow is negligible.

(e) Convection heat transfer and conduction heat transfer along the film (in the x-direction) is negligible.

(f) The transport properties of condensate are constant.

(g) No impurities in the vapour zone and condensate film.

(h) The temperature in the two-phase zone is uniform and maintained at $T_{\text {sat }}$.

(i) Condensation occurs only at the interface between the two-phase region and the saturated liquid region, $y=\delta_{l}$.

(j) Liquid phase pressure change in the $x$-direction is negligible, $\frac{\partial P_{l}}{\partial x}=0$.

(k) The change in capillary pressure is mainly due to the change in liquid pressure.

The governing equations for film condensation on an inclined plate embedded in a porous medium with the two-phase region effect are written in the following form.

At the interface of a two-phase zone with the liquid film, $y_{t}=0$ or $y_{l}=\delta_{l}$, the mass conservation equations can be written as

$\frac{\partial U_{l t}}{\partial x}+\frac{\partial V_{l t}}{\partial y_{t}}=0$

The density of the liquid is higher than vapour, $\rho_{l} \gg \rho_{v}$. Therefore, the assumption of a change in capillary pressure due to a change in liquid pressure is valid. Hence, the vapour pressure $P_{v}$ is approaching saturation $P_{\text {sat }}$ according to the Clausius-Clapeyron equation. Darcy's law gives relation between the velocity and the pressure gradient for film flow in a two-phase region by using the concepts of capillary pressure. Hence the liquid velocity in the two-phase region can be written as follows.

For the $x$-component velocity, the liquid propagates with gravitational effects, included to account for capillary action against an opposing force of gravity.

$U_{l t}=-\frac{K_{l}}{\mu_{l}} \frac{\partial P_{c}}{\partial x}+\frac{K_{l}\left(\rho_{l}-\rho_{v}\right) g}{\mu_{l}} \cos \phi$

For the $y$-component velocity, the liquid propagates with capillary effect only.

$V_{l t}=-\frac{K_{l}}{\mu_{l}} \frac{\partial P_{c}}{\partial y}$

where $P_{c}$ is the capillary pressure, defined as [31]

$P_{c}=\frac{\sigma}{\sqrt{K / \varepsilon}} j_{S}$ 
The non-dimensional function of non-dimensional saturation, $j_{s}$ is used to normalize capillary pressure data by considering the variations in porosity and permeability. It is also known as the Leverette J-function and can represented by the Leverett's [45] cubic polynomial of equation [34-35, 46-47].

$j_{S}=1.417(1-S)-2.120(1-S)^{2}+1.263(1-S)^{3}$

with the boundary condition of $S=1$ at liquid film thickness $\delta=0$.

The capillary pressure represented in the form of $P_{g}-P_{l}=P_{c}(S$, etc. $)$ can then be reduced to in a function of the non-dimensional saturation, $S$ only.

$P_{c}=P_{c}(S)$

$S$ is also the effective saturation or the normalized saturation, and defined as [34]

$S=\frac{S_{l}-S_{l i}}{1-S_{l i}}$

where $S_{l}$ is the wetting phase saturation and $S_{l i}$ is the minimum wetting phase saturation.

In the neighbourhood of the film surface $S$ is close to $1(S \rightarrow 1)$, Eq. (6) can be simplified by taking [8].

$j_{S}=\gamma(1-S)$

where $\gamma$ is the constant of proportionality that relates the Leverette J-function and the saturation.

Eq. (6) shows how the Leverett J-function (also known as the dimensionless function of saturation) varies with $y$ from $S=0$ (at the interface of a two-phase zone with vapour zone $y=\delta_{t}$ ) to $S=1$ (at the interface of a two-phase zone with liquid film $y=\delta_{l}$ ). Since the liquid film in a twophase region that near to the interface $y_{t} \cong 0$ (or $y \cong \delta_{l}$ ) is almost fully saturated, one can say that the saturation is approaching unity $(S \rightarrow 1)$. Hence the liquid saturation correlation at $y_{t} \cong 0$ (or $y \cong \delta_{l}$ ) can be approximated with a linear function expressed as

$S=\frac{y}{\delta_{t}}$

Differentiating Eq. (10) and get

$\frac{\partial S}{\partial y}=\frac{1}{\delta_{t}}$

The liquid phase permeability is given as

$K_{l}=K K_{r l}$

where $K$ is the absolute permeability or the permeability of the porous medium in single-phase flow, and $K_{r l}$ is the relative permeability of the liquid phase.

If $S$ close to $1(S \rightarrow 1)$, the liquid relative permeability is considered as a linear function of saturation and could be approximated as 
$K_{r l}=\xi S$

where $\xi$ is the constant of proportionality. By substituting Eq. (13) into Eq. (12), the liquid phase permeability can be re-written as

$K_{l}=\xi S K$

Since the two-phase zone is isothermal, and condensation occurs only at the interface between the two-phase region and the liquid saturated region $y_{t} \cong 0$ (or $y \cong \delta_{l}$ ), the mass balance equation for the mass of condensate at the interface of the liquid film with the two-phase zone $y=\delta_{l}$ can then be obtained as

$\frac{\partial}{\partial x} \int_{0}^{\delta_{l}} \rho_{l} U_{l} d y=\dot{m}_{\text {condensate }}^{\prime \prime}-\dot{m}_{\text {capillary }}^{\prime \prime}$

The energy balance which states that the rate of energy release due to condensation at the interface of a liquid film with the two-phase zone $y=\delta_{l}$ is equal to the heat conducted through the liquid film. Fourier's law is adequately described the conduction heat transfer in a liquid film.

$q^{\prime \prime}=\frac{\lambda}{\delta_{l}}\left(T_{s a t}-T_{w}\right)$

The mass flux of condensate can be represented as

$\dot{m}_{\text {condensate }}^{\prime \prime}=\frac{q^{\prime \prime}}{h_{f g}}$

Substituting Eq. (16) into Eq. (17), the mass flux of condensate can be re-written as

$\dot{m}_{\text {condensate }}^{\prime \prime}=\frac{\lambda}{\delta_{l} h_{f g}}\left(T_{\text {sat }}-T_{w}\right)$

However, not all the condensate flows down the plane by gravitational force. Due to capillary pressure, some portion of the condensate flows along the $y$-direction, which is perpendicular to the plate and causes the presence of a two-phase zone.

At the interface of a liquid film with a two-phase zone $\left(y=\delta_{l}\right)$, Eq. (4) the velocity in the $y$ direction could be re-written as follow according to Eq. (7) and Eq. (10).

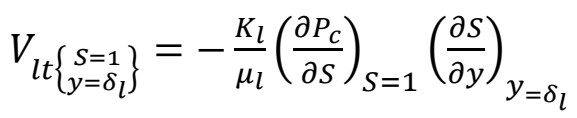

Referring to Eq. (6) and Eq. (14), both $j_{S}$ and $K_{l}$ are functions of $S$. Thus, the partial derivative of capillary pressure with respect to $S$ can be represented as

$\left(\frac{\partial P_{c}}{\partial S}\right)_{S=1}=\frac{\sigma}{\sqrt{K_{l} / \varepsilon}} \frac{\partial j_{S}}{\partial S}+j_{S} \frac{\varepsilon^{1 / 2}}{K_{l}^{3 / 2}} \frac{\partial K_{l}}{\partial S} \sigma$

Substituting Eq. (9), (11), (14) and (20) into Eq. (19), a simplified form of liquid velocity at the interface of a liquid film with two-phase zone $y=\delta_{l}$ due to the capillary force can be re-written as 


$$
V_{l t}\left\{\begin{array}{c}
S=1 \\
y=\delta_{l}
\end{array}\right\}=\frac{\gamma \sigma}{\mu} \sqrt{\xi K \varepsilon}\left(\frac{\partial S}{\partial y}\right)_{y=\delta_{l}}
$$

Hence the mass flux due to capillary force can be expressed as

$$
\dot{m}_{\text {capillary }}^{\prime \prime}=\frac{\rho_{l} \gamma \sigma}{\mu \delta_{t}} \sqrt{\xi K \varepsilon}
$$

The film thickness is small compared to the plate length, $\delta \ll L$. The liquid pressure in the $x$ direction is negligible as suggested by many previous researchers [34-35, 46]. The change in capillary pressure is mainly due to the change in liquid pressure. Hence the capillary effect in the $\mathrm{x}$-direction is negligible, $\partial P_{c} / \partial x=0$. Also, $K_{l}=\xi S K$ and $S=1$ at the interface of the liquid film with a twophase zone, $y=\delta_{l}$, the liquid film velocity can be represented as

$U_{l} \approx\left(\rho_{l}-\rho_{v}\right) g \frac{\xi K}{\mu} \cos \phi$

By substituting Eq. (17)-(18), (23) into Eq. (15) and integrating over $y$, the mass balance equation for the mass of condensate at the interface of a liquid film with two-phase zone $y=\delta_{l}$ can be rewritten as

$\frac{\partial \delta_{l}}{\partial x}=\frac{B}{\delta_{l}}-\frac{A}{\delta_{t}}$

where

$A=\frac{\gamma \sigma}{\left(\rho_{l}-\rho_{v}\right) g \cos \phi} \sqrt{\frac{\varepsilon}{\xi K}}$

$B=\frac{\mu \lambda}{\rho_{l}\left(\rho_{l}-\rho_{v}\right) g \cos \phi K \xi h_{f g}}\left(T_{s a t}-T_{w}\right)$

Eq. (24) represents the liquid film thickness change along the plate.

Since liquid is sucked from the liquid film across the interface of the liquid film and two-phase zone, so the liquid flow is due to the capillary force in y-direction only. Hence, the mass flow rate of condensate at the interface of a two-phase zone with the liquid film, $y_{t}=0$ is represented as

$\frac{\partial}{\partial x} \int_{y_{t}=0}^{y_{t}=\delta_{t}}\left(\rho_{l}-\rho_{v}\right) U_{l} d y=\dot{m}_{\text {capillary }}^{\prime \prime}$

In two phase zone, the free and wetting films formed by sucking the liquid away from the liquid film pass across an interface of liquid film with two phase zone $y=\delta_{l}$ (or $y_{t}=0$ ). Hence the Eq. (26) could be rewritten as

$\frac{\partial}{\partial x} \int_{y_{t}=0}^{y_{t}=\delta_{t}} U_{l} d y=-\frac{K_{l}}{\mu_{l}}\left(\frac{\partial P_{c}}{\partial S}\right)_{S=1}\left(\frac{\partial S}{\partial y}\right)_{y_{t}=0}$

where $y_{t}=0$ is at the interface of a liquid film with a two-phase zone, $y=\delta_{l}$. 
The liquid saturation is unity, $S=1$ at the interface of the two-phase zone and liquid film, $y_{t}=$ 0 . Substituting Eq. (11), (20) and (23) into Eq. (28), the resulting expression is rearranged to get $\frac{\partial}{\partial x} \int_{y_{t}=0}^{y_{t}=\delta_{t}} \frac{y}{\delta_{t}} d y=\frac{A}{\delta_{t}}$

Solve Eq. (29) and substitute into Eq. (24), the film thickness varying in the x-direction at liquid film could be rewritten in the following form

$\frac{d \delta_{l}}{d x}=\frac{B}{\delta_{l}}-\frac{\sqrt{A}}{2 \sqrt{x}}$

Based on the structure of Eq. (30) one shall seek the solution of Eq. (30) in the form

$\delta_{l}=c \sqrt{x}$

Substituting Eq. (31) into Eq. (30) find $c$ as follows

$C=\frac{-\sqrt{A}+\sqrt{A+8 B}}{2}$

Substitute Eq. (25)-(26) into Eq. (32) and solve for Eq. (31) yields

$\delta_{l}=\frac{\sqrt{x}}{2}\left\{-\left[\frac{\gamma \sigma}{\left(\rho_{l}-\rho_{v}\right) g \cos \phi}\left(\frac{\varepsilon}{\xi K}\right)^{\frac{1}{2}}\right]^{\frac{1}{2}}+\left[\frac{\gamma \sigma}{\left(\rho_{l}-\rho_{v}\right) g \cos \phi}\left(\frac{\varepsilon}{\xi K}\right)^{\frac{1}{2}}+\frac{8 \mu \lambda}{\rho_{l}\left(\rho_{l}-\rho_{v}\right) g \cos \phi K \xi h_{f g}}\left(T_{s a t}-T_{w}\right)\right]^{\frac{1}{2}}\right\}$

From the heat balance equation,

$\alpha=\frac{\lambda}{\mathrm{c} \sqrt{x}}$

one obtains

$N u=\frac{\sqrt{x}}{c}$

Consequently, the local Nusselt number is

$N u=2 \sqrt{x}\left\{-\left[\frac{\gamma \sigma}{\left(\rho_{l}-\rho_{v}\right) g \cos \phi}\left(\frac{\varepsilon}{\xi K}\right)^{\frac{1}{2}}\right]^{\frac{1}{2}}+\left[\frac{\gamma \sigma}{\left(\rho_{l}-\rho_{v}\right) g \cos \phi}\left(\frac{\varepsilon}{\xi K}\right)^{\frac{1}{2}}+\frac{8 \mu \lambda}{\rho_{l}\left(\rho_{l}-\rho_{v}\right) g \cos \phi K \xi h_{f g}}\left(T_{s a t}-T_{w}\right)\right]\right\}^{-1}$

In the criterion form, the average Nusselt number for steady-state heat transfer within the twophase zone of a porous layer on an inclined plate can be represented as

$\overline{N u}=\frac{1}{-\sqrt{\frac{\gamma \sqrt{K} 1}{16 \xi L B o}}+\sqrt{\frac{\gamma \sqrt{K} 1}{16 \xi L B o}+\frac{1}{2 \xi A r^{*} P r \mathrm{~K}_{\mathrm{u}}}}}$ 
The parameters $\xi$ and $\gamma$ are estimated from experimental data. $\xi$ is taken to be 1. $\gamma$ estimated from the Leverett J-function. The constant $\gamma$ is determined by using trial and error analysis method. It is found that $\gamma=0.025$ best matches to the experimental data.

\section{Results}

Without the two-phase region effect, $B o \gg 1$, the term $\frac{\gamma}{16 \xi} \frac{\sqrt{K}}{L} \frac{1}{B o}$ in Eq. (37) is negligible. By substituting $\xi=1$, the Eq. (37) can be reduced to the classical solution of Eq. (1) for film condensation in porous media, $\overline{N u}=\left(2 A r^{*} \operatorname{PrK}_{\mathrm{u}}\right)^{\frac{1}{2}}$. The Nusselt number as a function of $A r^{*} \operatorname{PrK}_{\mathrm{u}}$ with porosity $\varepsilon=0.38$, permeability $K=5.075 \times 10^{-4} \mathrm{~mm}^{2}, \gamma=1.4$ and $\beta=1$ for different $B o$ number is shown in Figure 2. For a very large Bo number corresponds to no two-phase region effect, gives a linear line on the graph. The curve is getting closer to the no two-phase effect line when Bo number is getting bigger. The capillary force significantly improves the heat transfer rate, particularly for large $A r^{*} \operatorname{Pr} \mathrm{K}_{\mathrm{u}}$ number. As the Bo number increases, the capillary needs a greater $A r^{*} \operatorname{Pr} \mathrm{K}_{\mathrm{u}}$ number for the action to begin. For modest $A r^{*} \operatorname{Pr} \mathrm{K}_{\mathrm{u}}$ number, the capillary force shows very little effect and could say the effect is negligible. The line starts to deviate upward from the line with no two-phase effect.

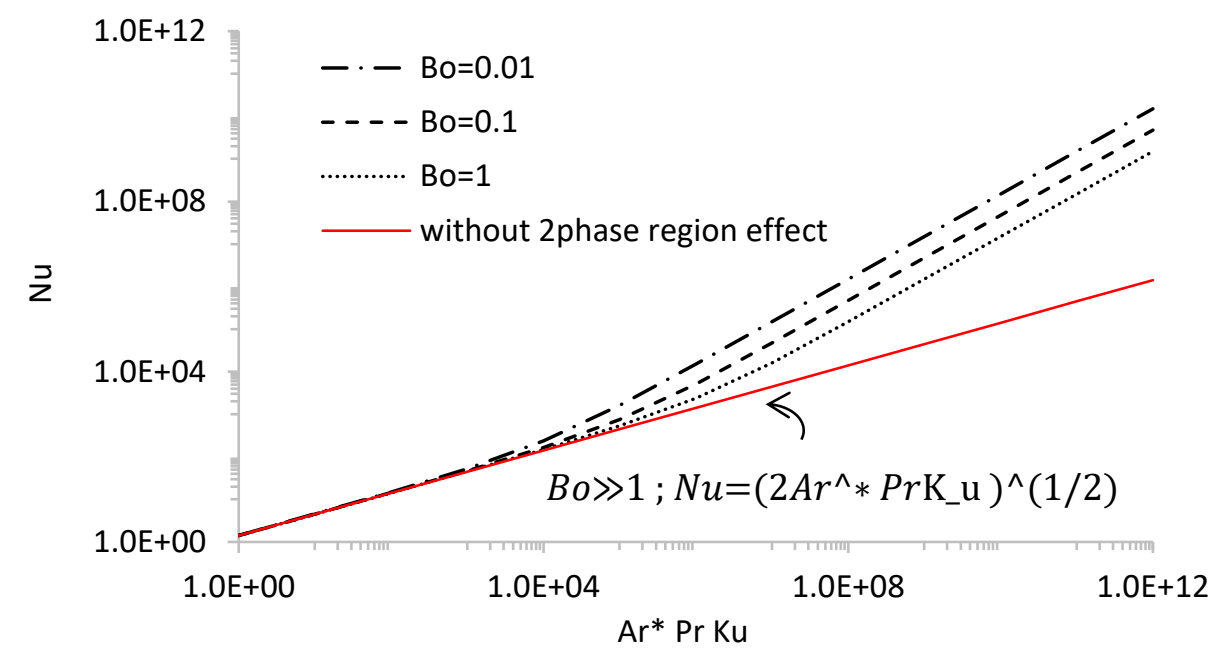

Fig. 2. Nusselt number as function of $\boldsymbol{A r}^{*} \operatorname{Pr}_{\boldsymbol{u}}$ for $\boldsymbol{\varepsilon}=0.38, \mathrm{~K}=0.0005075$ $\mathrm{mm}^{2}, \gamma=0.025$ and $\xi=1$

Figure 3 and 4 show how the capillary force affects the condensation heat transfer by Kutateladze number $\mathrm{K}_{\mathrm{u}}$. The larger the Kutateladze number, the higher the heat transfer rate. In terms of thermal resistance, the temperature drop across any film layer is proportional to the thermal resistance of that layer if the heat flux is remaining constant. When heat is transferred through a low resistance film layer, the temperature drop is lesser. Consequently, the Kutateladze number is high and causes a high heat transfer rate.

It can also be seen from Figure 4 that the smaller the Bond number, the higher the heat transfer rate. The smaller the Bond number, the stronger the surface tension force. Hence, more liquid will be sucked up to the two-phase region making liquid film thinner and causing a steeper temperature gradient in the liquid zone resulting in the higher heat transfer rate for the smaller Bond number. 


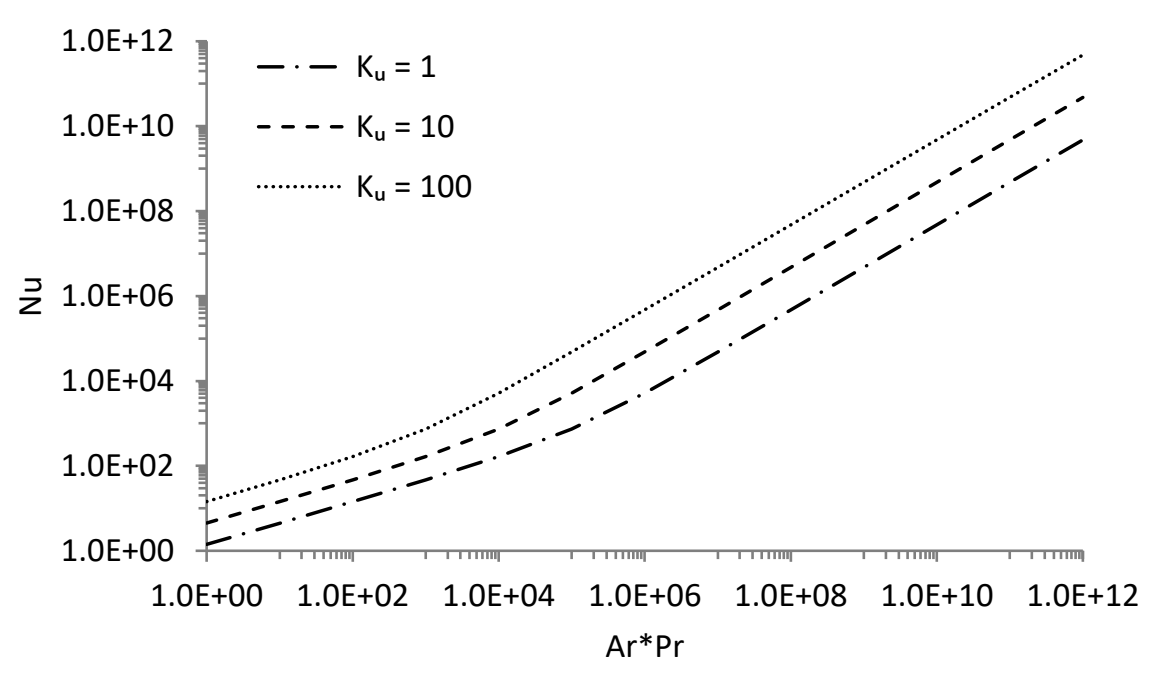

Fig. 3. The effect of Kutateladze number, $\boldsymbol{K}_{\boldsymbol{u}}$

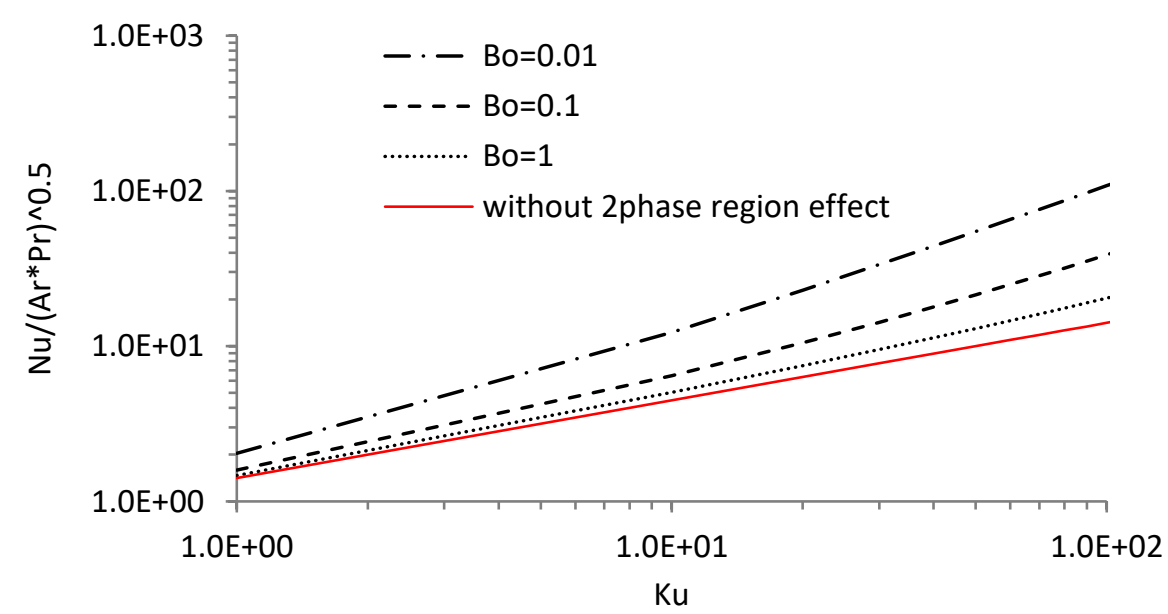

Fig. 4. The condensation heat transfer results for porosity $\varepsilon=0.38$, permeability $\mathrm{K}=0.0005075 \mathrm{~mm}^{2}, \gamma=0.025$ and $\xi=1$

Figure 5 shows the comparison between Majumdar and Tien's [34] model, Plumb's [33] model, present model, and experimental results of Dvorovenko [18] with no two-phase region effect. The results show a good agreement between the predictions of the present model to the experimental data. Whereas, the model developed by Plumb [33], and Majumdar and Tien [34] underpredicts the heat transfer coefficient of this zone and hence shows a lower heat transfer rate.

Figure 6 shows the comparison of experimental results with the present model and theoretical model developed by Nakoryakov and Gorin [8]. Among the numerous data of Dvorovenko [18], the sample of $d=0.8 \mathrm{~mm}, L=250 \mathrm{~mm}, \cos \phi=0.10343$ was selected for further investigation as it deviates far above the solution by Nakoryakov and Gorin [8]. It means the selected sample has a higher heat transfer rate compared to other samples which might be due to the presence of a twophase region effect. 


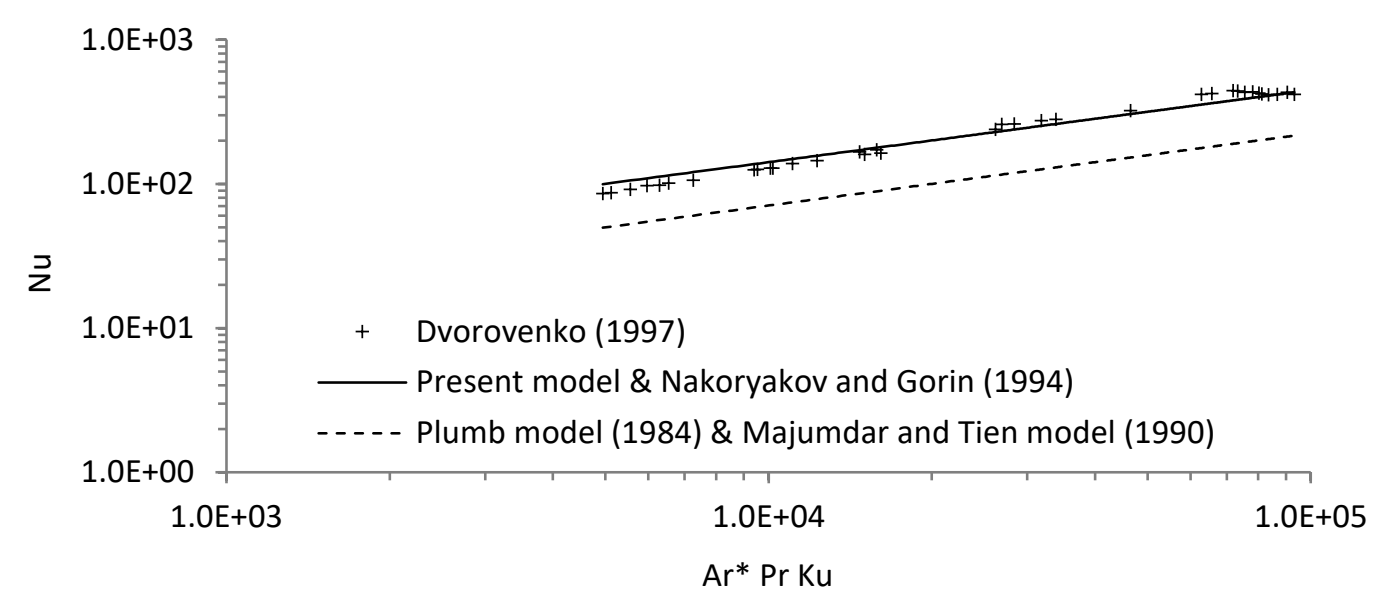

Fig. 5. Comparison of the predicted model developed by Majumdar and Tien [34], Plumb [33] to the present model (with $\xi=1$ ) and experimental data with no twophase region effect $(\boldsymbol{B o} \gg \mathbf{1})$ for film condensation on an inclined plate with a length of $500 \mathrm{~mm}$ embedded in porous media with a grain size of $800 \mu \mathrm{m}$

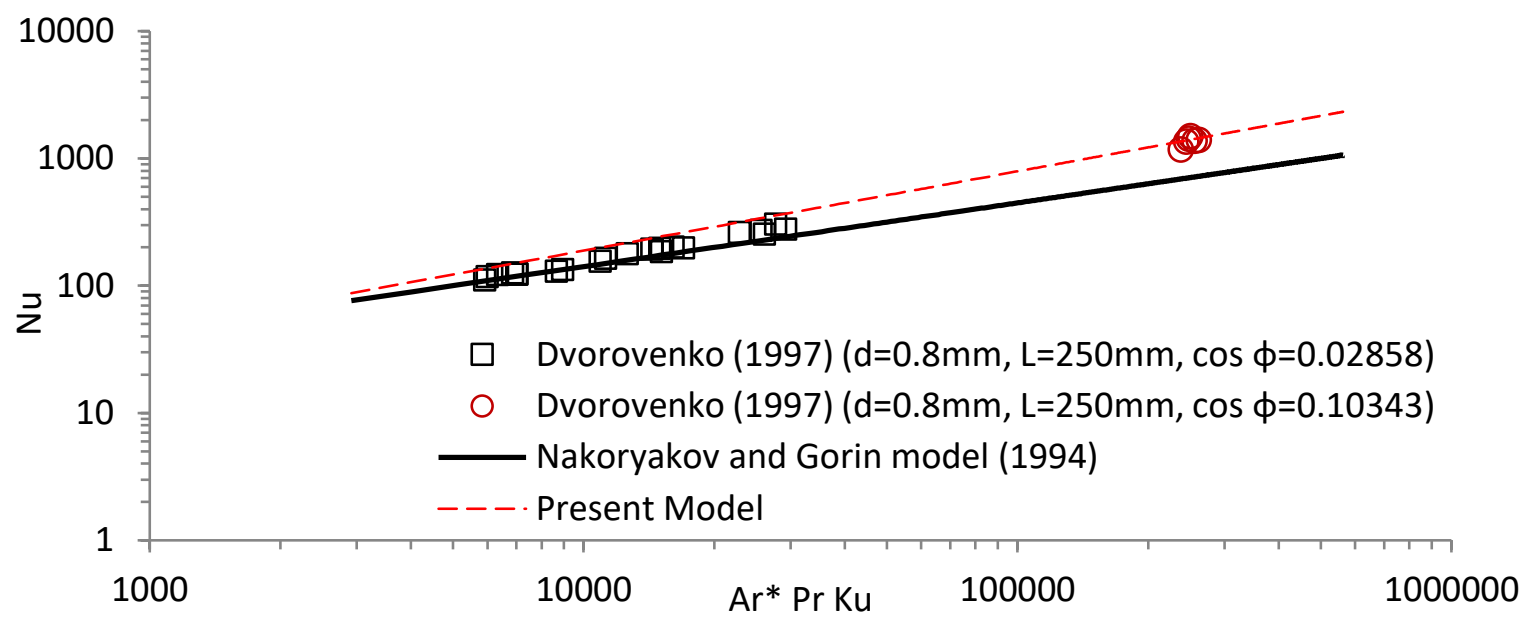

Fig. 6. Comparison of experimental results with the theoretical model for film condensation on an inclined plate embedded in porous media with a grain size of $800 \mu \mathrm{m}$ and plate length of $250 \mathrm{~mm}$

This selected sample was further investigated and found it has a relatively small temperature difference between the plate wall temperature $T_{w}$ and saturation temperature $T_{s a t}$, which is less than $4^{\circ} \mathrm{C}$ if compared to other samples. Besides, it has the thinnest liquid film thickness about half the grain size with $\delta=0.45 \mathrm{~mm}$ approximately, and the gradient of the slope (or the thermal resistance) is the smallest. While for other samples, the liquid film thickness is thicker than the grain size. This finding has clarified Chang's research finding in which the heat transfer rate is higher for the sample with a two-phase region effect. It is due to more liquid condensate into the two-phase region, causing the thinning of liquid film thickness and gives a sharper temperature gradient in the liquid zone [37].

A comparison between the present two-phase model, experimental results [18], and some other two-phase models developed by Cheng [9], Plumb [33], and Majumdar and Tien [34] is showed in Figure 7.

The two-phase model developed by Majumdar and Tien [34] and Plumb [33] had have rearranged to ease the comparison. Both models employed the same parameters used in the current study and represented as 
Majumdar and Tien's model

$N u_{L}=\left[-\sqrt{0.373^{2} \frac{\sqrt{K}}{L} \frac{1}{B o}}+\sqrt{0.373^{2} \frac{\sqrt{K}}{L} \frac{1}{B o}+\frac{2}{A r^{*} P r K_{\mathrm{u}}}}\right]^{-1}$

Plumb's model

$$
N u_{L}=\left[-\sqrt{\frac{j_{S}}{6(1+2 \Lambda)} \frac{\sqrt{K}}{L} \frac{1}{B o}}+\sqrt{\frac{j_{S}}{6(1+2 \Lambda)} \frac{\sqrt{K}}{L} \frac{1}{B o}+\frac{2}{A r^{*} \operatorname{PrK}_{\mathrm{u}}}}\right]^{-1}
$$

where $\Lambda$ is the parameter relating the saturation and capillary pressure. According to Brooks and Corey [48], $\Lambda$ will be in the range of $2 \leq \Lambda \leq 8$ for a variety of unconsolidated porous materials.

The graph of both numerical and experimental results for film condensation on an inclined plate embedded in porous media with a grain size of $800 \mu \mathrm{m}$ and a plate length of $250 \mathrm{~mm}$ presented in terms of the dimensionless parameters $\left(N u, A r^{*}, \operatorname{Pr}, \mathrm{K}_{\mathrm{u}}\right)$ plotted as shown in Figure 7. The experimental result shows a good agreement with the present model. The parameter of $\gamma$ in the present model was adjusted and found the best match with experimental data is by substituting $\gamma=$ 0.025 while $\xi$ would always equal to 1 as mentioned above.

The present, Plumb [33], and Majumdar and Tien [34] models all have a higher heat transfer coefficient than the model developed by Nakoryakov and Gorin [8]. In Nakoryakov and Gorin's [8] model, the two-phase region effect not taken into consideration, and the pure vapour assumed to be in direct contact with the liquid film. Whereas, the heat transfer coefficient of Chang's [37] model is very much lower than the model developed by Nakoryakov and Gorin [8] but could improve by adjusting its constant coefficient of 1.23 to a higher value.

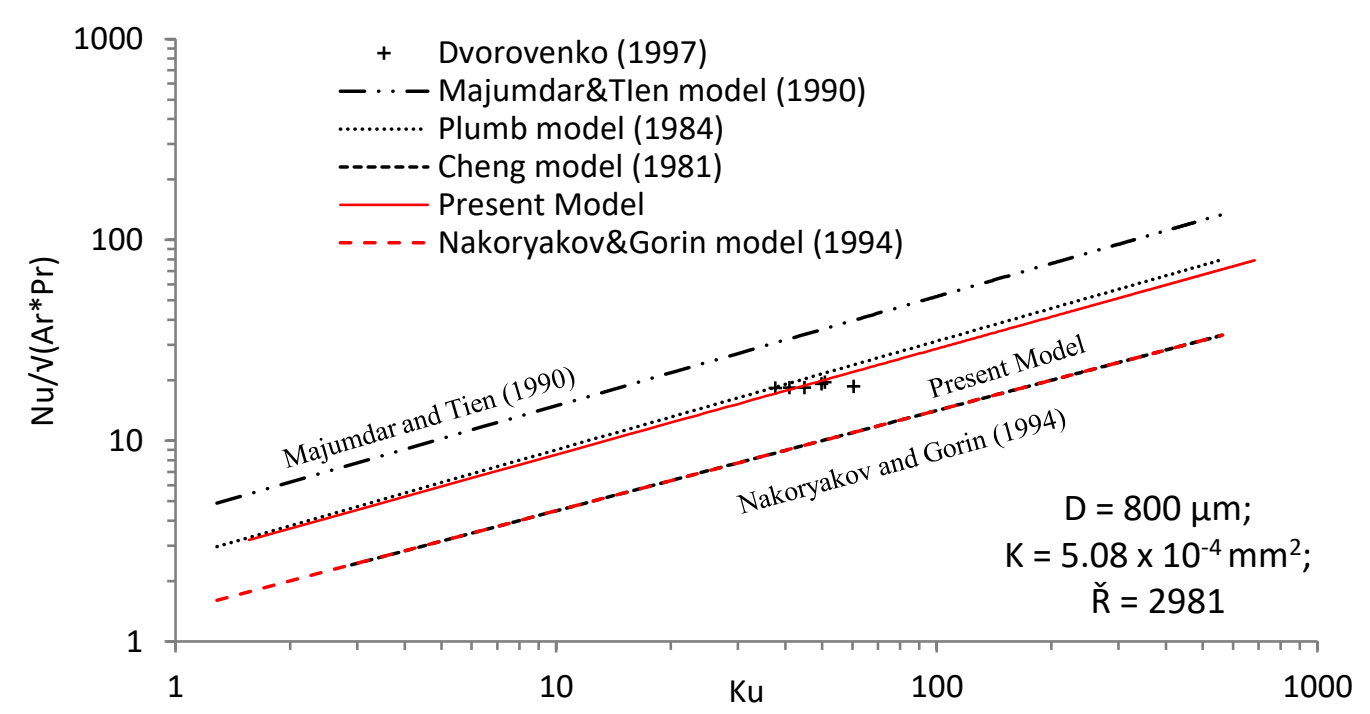

Fig. 7. Film condensation on an inclined plate with inclination angle of $\cos \varphi=$ 0.10343 and length of $250 \mathrm{~mm}$ embedded in porous media with grain size of $800 \mu \mathrm{m}$

Furthermore, the present model compared with the experimental results presented by Chung et al., [35] also the model developed by Cheng [9], Plumb [33], Majumdar and Tien [34], Chung et al., [35] and Nakoryakov and Gorin [8]. The condensation heat transfer rate represented by $\frac{\mathrm{Nu}}{\sqrt{A r^{*} P r}}$ versus 
Kutateladze number for film condensation embedded in porous media with glass beads size of 900 $\mu \mathrm{m}$ is showed in Figure 8. It can be seen that the model developed by Cheng [9] and Nakoryakov and Gorin [8] has a less steep line and is far below the models that account for the two-phase region effect as they did not include the two-phase region effect.

The local Nusselt number of Cheng's model [9] gives the heat transfer coefficient at a particular point on the heat-transfer surface expressed in the following equation.

$N u_{x}=\left[\frac{(2 n+1) R a_{x, L}}{2 J a}\right]^{\frac{1}{2}}$

where, $R a_{x, L}$ is the local Rayleigh number and $J a$ is the Jakob number while $n=0$ for wedge and $n=1$ for cone. Eq. (40) is applied by Chung et al., [35] to compare with their experimental results. By substituting $n=0$, represent $R a_{x, L}$ as $A r^{*} P r$, and $J a$ as $1 / \mathrm{K}_{\mathrm{u}}$, Eq. (40) becomes

$N u_{x}=\left[\frac{A r^{*} \operatorname{Pr} \mathrm{K}_{\mathrm{u}}}{2}\right]^{\frac{1}{2}}$

From Eq. (41), the average Nusselt number is $\overline{N u}=\left(2 A r^{*} \operatorname{Pr} \mathrm{K}_{\mathrm{u}}\right)^{\frac{1}{2}}$, which coincides with the solution by Nakoryakov and Gorin [8].

As seen in Figure 8, the experimental results of Chung et al., [35] are far below the two-phase model developed by Majumdar and Tien [34], Plumb [33], and present model. Conversely, the experimental results have a better agreement with the solution of film condensation developed by Nakoryakov and Gorin [8] and Cheng [9]. The heat transfer rate from the Majumdar and Tien's model is higher than the Plumb's model for all three glass beads size investigated by Chung et al., [35]. According to Majumdar and Tien [34], this is because of the Plumb's model under-predicts the size and liquid flux in the two-phase zone.

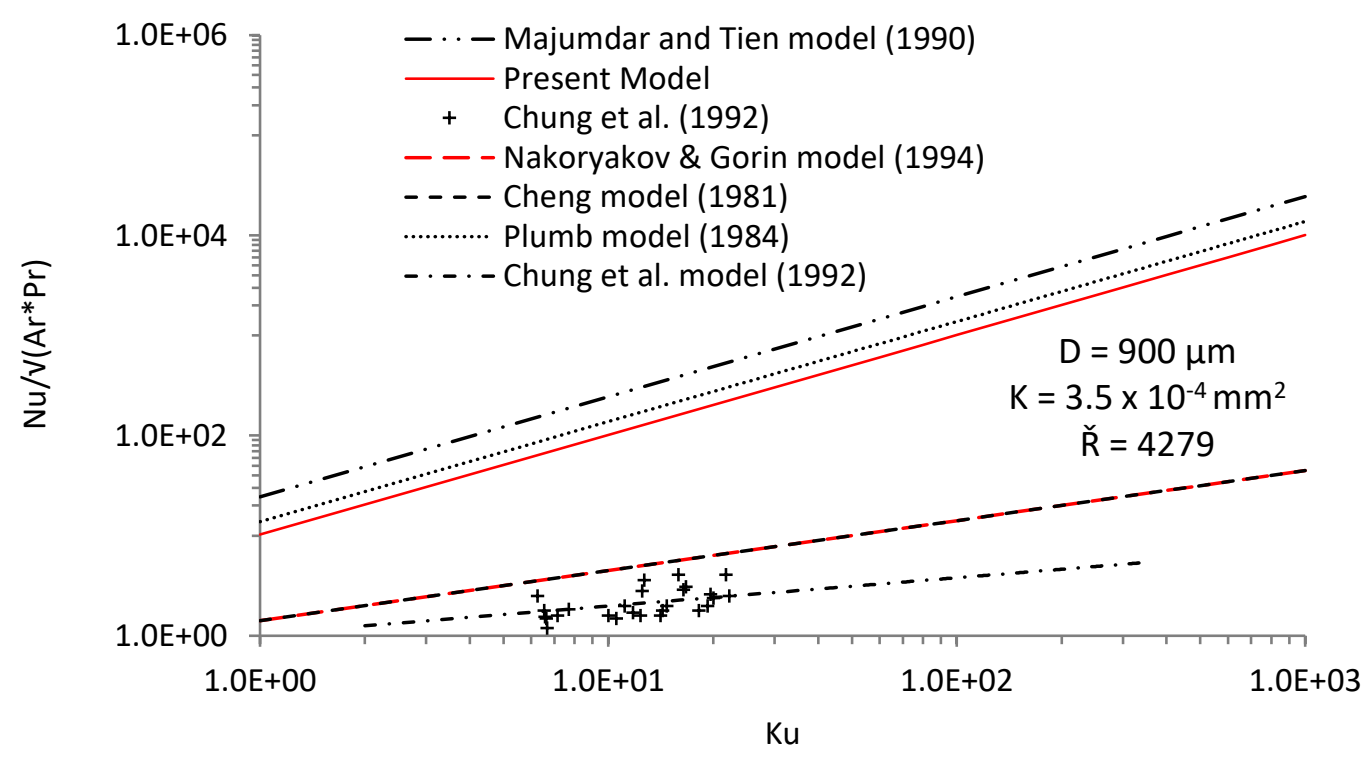

Fig. 8. Film condensation embedded in porous media with glass beads size of $900 \mu \mathrm{m}$

Chung et al., [35] account for the Non-Darcian and the capillary effects in their model. Although their results have shown to match with experimental results, the results did not show a good representation of liquid film condensation in a porous medium with the two-phase region effect. The 
experimental results obtained by Chung et al., [35] were much closer to the models developed by Nakoryakov and Gorin [8] and Cheng [9]. Their models had well-predicted liquid film condensation in porous media. Plumb [49] also indicated that the physical models by Chung et al., [35] and Cheng [9] get very similar results for the condensation heat transfer rate.

Other than that, Wang and Beckermann [47] said it is inappropriate to use the experimental results of Chung et al., [35] for two-phase models validation because the local film thickness in their experiments was about of the same order as the glass bead diameters. Kaviany [23] also stated that for local film thickness that comparable to particle size, the local volume averaged treatments is no longer valid as it breaks the continuum treatment. From the experimental results by White and Tien [16], they also could not conclude that the two-phase zone was present as the porous sample used had a dimension of $1.6 \mathrm{~cm} \times 1.6 \mathrm{~cm}$ only. This small area might not be sufficient for a two-phase zone to fully developed. Moreover, there is no temperature profile along the porous layer thickness presented by White and Tien [16] and Chung et al., [35]. There is no measurement of temperature distribution across the porous layer thickness in their experiment. Hence the results obtained could not prove that the isothermal two-phase region did exist.

From Figure 8, one can also see that both the experimental results and model by Chung et al., [35] give similar trends as the model by Nakoryakov and Gorin [8] and Cheng [9] but slightly lower. Hence, the finding reveals additional evidence of the experimental results by Chung et al., [35] that the results might not have the two-phase region effect. As a result, the validity of the developed twophase model has verified through the experimental results of Dvorovenko [18]. As shown in Figure 7, the results indicate a good agreement between the experimental result and the present model of Eq. (37). Herein, the study has successfully demonstrated a further systematic study of Nakoryakov and Gorin [8] by representing the heat transfer results in a non-dimensional function of $A r^{*}, P r, \mathrm{~K}_{\mathrm{u}}$ and $B o$. With this, the present model can be applied to determine the film condensation in a heat pipe, which is a simple yet effective capillary two-phase heat transfer device. A simple Nusselt correlation will ease the engineers in designing the heat pipe wick structure and monitoring of heat pipe performance. It allows engineers to predict and control "dry-outs" in the evaporative section of heat pipes by estimating the condensation rates in the condenser section. Subsequently, this could lead to cost savings in the design and construction of heat pipes.

\section{Conclusions and Recommendations}

A simple physico-mathematical model of film condensation on an inclined plate embedded in a porous medium with a two-phase region effect is herein developed and presented with the Leverette J-function prediction model, resulting in an easier calculation and results that are more representative. In the two-phase region, the capillary pressure and relative permeability are the key parameters governing the fluid flow in a porous medium. Taking the capillary effects into consideration results in higher heat transfer and condensation rates. Also, the presence of the twophase region causes the liquid film thickness to become thin. Therefore, the smaller the Bo number, the stronger the surface tension force, the higher the heat transfer rate. From the results obtained, numerical and experimental results are in similar trends. The Nusselt number increases when $A r^{*} \operatorname{Pr} \mathrm{K}_{\mathrm{u}}$ increases. The present model is proved to give comparable results with the experimental data. The parameter of $\gamma$ in the present model was determined. It is found that the present model best match with experimental data by substituting $\gamma=0.025$ and $\xi=1$. However, there are limited experimental investigations on film condensation embedded in porous media with the two-phase region effect conducted over the last two decades ago. Since technology has progressed in every field imaginable, experimental verification for film condensation with two-phase region effect could be 
possibly done today in more detail, precise and accurate with the use of advanced equipment and software. Other than that, the present model could include the effect of inertial and vapour flow as well as consider thin liquid film near the plate wall and non-Darcian flow. With these effects and considerations could allow for true prediction of heat transfer and condensation rate. Nevertheless, the present model with the Leverette J-function prediction formula takes into consideration the twophase region effect is easier to use and more convenient compared with the earlier formula. Moreover, the calculated results are more representative. Hence, enable the engineers and designers to obtain the condensation heat transfer coefficient easily; evaluate the rates of condensation heat transfer to control "flooding" in the condensation section of heat pipes.

\section{References}

[1] Nusselt, Wilhelm. "Die oberflachenkondensation des wasserdamphes." VDI-Zs 60 (1916): 541.

[2] Sparrow, Ephraim M., and John L. Gregg. "A boundary-layer treatment of laminar-film condensation." Journal of Heat Transfer 81, no. 1 (1959): 13-18. https://doi.org/10.1115/1.4008118

[3] Chen, Michael Ming. "An analytical study of laminar film condensation: part 1-flat plates." Journal of Heat Transfer (1961): 48-54.

https://doi.org/10.1115/1.3680467

[4] Koh, J. C. Y., Ephraim M. Sparrow, and J. P. Hartnett. "The two-phase boundary layer in laminar film condensation." International Journal of Heat and Mass Transfer 2, no. 1-2 (1961): 69-82. https://doi.org/10.1016/0017-9310(61)90015-1

[5] Dhir, Vijay, and John Lienhard. "Laminar film condensation on plane and axisymmetric bodies in nonuniform gravity." Journal of Heat Transfer (1971): 97-100. https://doi.org/10.1115/1.3449773

[6] Merte Jr, Herman. "Condensation heat transfer." In Advances in Heat Transfer, vol. 9, pp. 181-272. Elsevier, 1973. https://doi.org/10.1016/S0065-2717(08)70063-7

[7] Rose, J. W. "Fundamentals of condensation heat transfer: laminar film condensation." JSME international journal. Ser. 2, Fluids engineering, heat transfer, power, combustion, thermophysical properties 31, no. 3 (1988): $357-375$. https://doi.org/10.1299/jsmeb1988.31.3 357

[8] V. E. Nakoryakov and A. V. Gorin. "Heat and mass transfer in two-phase systems." Institute of Thermophysics, Siberian Branch of Russian Academy of Sciences, Novosibirsk, 1994.

[9] Cheng, Ping. "Film condensation along an inclined surface in a porous medium." International Journal of Heat and Mass Transfer 24, no. 6 (1981): 983-990. https://doi.org/10.1016/0017-9310(81)90129-0

[10] Sparrow, E. M., and R. D. Cess. "Free convection with blowing or suction." (1961): 387-389. https://doi.org/10.1115/1.3682298

[11] Parmentier, E. M. "Two phase natural convection adjacent to a vertical heated surface in a permeable medium." International Journal of Heat and Mass Transfer 22, no. 6 (1979): 849-855. https://doi.org/10.1016/0017-9310(79)90025-5

[12] Hong, J. T., C. L. Tien, and M. Kaviany. "Non-Darcian effects on vertical-plate natural convection in porous media with high porosities." International journal of heat and mass transfer 28, no. 11 (1985): 2149-2157. https://doi.org/10.1016/0017-9310(85)90109-7

[13] Tong, T. W., and E. Subramanian. "A boundary-layer analysis for natural convection in vertical porous enclosuresuse of the Brinkman-extended Darcy model." International journal of heat and mass transfer 28, no. 3 (1985): 563571. https://doi.org/10.1016/0017-9310(85)90179-6

[14] M. Kaviany. "Boundary-layer treatment of film condensation in the presence of a solid matrix," International Journal of Heat and Mass Transfer 29, no. 6 (1986): 951-954. https://doi.org/10.1016/0017-9310(86)90191-2

[15] White, S. M., and C. L. Tien. "Analysis of laminar film condensation in a porous medium." In asme. 1987.

[16] White, S. M., and C. L. Tien. "An experimental investigation of film condensation in porous structures." ihp (1987).

[17] Renken, K. J., M. Aboye, M. Carneiro, and K. Meechan. "Effect of vapor velocity on film condensation along a surface embedded in a porous medium." International communications in heat and mass transfer 20, no. 1 (1993): 1-13. https://doi.org/10.1016/0735-1933(93)90002-D 
[18] I. V. Dvorovenko. "Heat Transfer in Film Condensation on a Plate Embedded in a Packed Bed." PhD Thesis, Kuzbass State Technical University, Kemerovo, Russia, 1997.

[19] Dorokhov, A. R., V. S. Loginov, Irina Petrovna Ozerova, P. T. Petrik, A. R. Bogomolov, and I. V. Dvorovenko. "Transient heat transfer during steam film condensation on a horizontal tube." Thermal engineering 44, no. 3 (1997): $244-246$.

[20] Al-Nimr, M. A., and M. K. Alkam. "Film condensation on a vertical plate imbedded in a porous medium." Applied energy 56, no. 1 (1997): 47-57. https://doi.org/10.1016/S0306-2619(96)00022-0

[21] Masoud, S. A., M. A. Al-Nimr, and M. K. Alkam. "Transient film condensation on a vertical plate imbedded in porous medium." Transport in Porous Media 40, no. 3 (2000): 345-354.

https://doi.org/10.1023/A:1006606426819

[22] Todd, D. K., and L. W. Mays. "Groundwater Hydrology, II Third Edition. John Wiley and Sons, Inc." (2005).

[23] Kaviany, Maasoud. Principles of heat transfer in porous media. Springer Science \& Business Media, 2012.

[24] R. H. Nilson and L. A. Romero. "Self-Similar Condensing Films in Porous Media." International Journal of Heat and Mass Transfer 23 (1980): 1461-1470. https://doi.org/10.1016/0017-9310(80)90150-7

[25] P. Cheng and D. K. Chui. "Transient film condensation on a vertical surface in a porous medium." International Journal of Heat and Mass Transfer 27, no. 5 (1984): 795-798. https://doi.org/10.1016/0017-9310(84)90149-2

[26] D. Poulikakos and J. Orozco. "A Study of Condensation on a Vertical Internally Cooled Pipe Embedded in a Porous Medium." International Communications in Heat and Mass Transfer 13, (1986): 181-192. https://doi.org/10.1016/0735-1933(86)90058-8

[27] H. H. Bau and K. E. Torrance. "Boiling in low permeability porous materials." International Journal Heat and Mass Transfer 25, no. 1 (1982): 45-55. https://doi.org/10.1016/0017-9310(82)90233-2

[28] C. H. Sondergeld and D. L. Turcotte. "An experimental study of two-phase convection in a porous medium with applications to geological problems." Journal of Geophysical Research 82, no. 14 (1977): 2045-2053. https://doi.org/10.1029/JB082i014p02045

[29] T. Abe, E. R. G. Eckert, and R. J. Goldstein. "A parametric study of boiling in a porous bed." Wärme - und Stoffübertragung 16, no. 2 (1982): 119-126. https://doi.org/10.1007/BF01459769

[30] V. Dhir and I. Catton. "Dryout heat fluxes for inductively heated particulate beds." Journal of Heat Transfer 99, no. 2 (1977): 250-256. https://doi.org/10.1115/1.3450677

[31] K. S. Udell. "Heat transfer in porous media heated from above with evaporation, condensation, and capillary effects." ASME J. Heat Transfer 105, no. 2 (1983): 485-492. https://doi.org/10.1115/1.3245611

[32] K. S. Udell. "Heat transfer in porous media considering phase change and capillarity-the heat pipe effect." International Journal of Heat and Mass Transfer 28, no. 2 (1985): 485-495. https://doi.org/10.1016/0017-9310(85)90082-1

[33] PLUMB, O. "Capillary effects on film condensation in porous media." In 19th Thermophysics Conference, p. 1789. 1984. https://doi.org/10.2514/6.1984-1789

[34] A. Majumdar and C. L. Tien. "Effects of Surface Tension on Film Condensation in a Porous Medium." Journal of Heat Transfer 112, no. 3 (1990): 751-757. https://doi.org/10.1115/1.2910450

[35] J. N. Chung, O. A. Plumb, and W. C. Lee. "Condensation in a Porous Region Bounded by a Cold Vertical Surface." Journal of Heat Transfer 114, no. 4 (1992): 1011-1018. https://doi.org/10.1115/1.2911871

[36] L. Bridge, R. Bradean, M. J. Ward, and B. R. Wetton. "The analysis of a two-phase zone with condensation in a porous medium." Journal of Engineering Mathematics 45, no. 3 (2003): 247-268. https://doi.org/10.1023/A:1022690802938

[37] T.-B. Chang. "Laminar Film Condensation on a Horizontal Plate in a Porous Medium with Surface Tension Effects." Journal of Marine Science and Technology 13, no. 4 (2005): 257-264.

[38] D. Jafari, P. Di Marco, S. Filippeschi, and A. Franco. "An experimental investigation on the evaporation and condensation heat transfer of two-phase closed thermosyphons." Experimental Thermal and Fluid Science 88 (2017): 111-123.

https://doi.org/10.1016/i.expthermflusci.2017.05.019 
[39] Rohsenow, Warren M., James P. Hartnett, and Young I. Cho. Handbook of heat transfer. Vol. 3. New York: McGrawHill, 1998.

[40] H. Hashimoto and F. Kaminaga. "Heat transfer characteristics in a condenser of closed two-phase thermosyphon: Effect of entrainment on heat transfer deterioration." Heat Transfer-Asian Research: Co-sponsored by the Society of Chemical Engineers of Japan and the Heat Transfer Division of ASME, 31, no. 3 (2002): 212-225. https://doi.org/10.1002/htj.10030

[41] H. Jouhara and A. J. Robinson. "Experimental investigation of small diameter two-phase closed thermosyphons charged with water, FC-84, FC-77 and FC-3283." Applied Thermal Engineering 30, no. 2 (2010): 201-211. https://doi.org/10.1016/i.applthermaleng.2009.08.007

[42] Q. Song, G. Chen, Z. Yang, H. Wang, and M. Gong. "New adiabatic and condensation two-phase flow pattern maps of R14 in a horizontal tube." International Journal of Heat and Mass Transfer 127 (2018): 910-924. https://doi.org/10.1016/i.ijheatmasstransfer.2018.07.129

[43] Q. Song, G. Chen, H. Xue, Y. Zhao, and M. Gong. "R14 flow condensation heat transfer performance: Measurements and modeling based on two-phase flow patterns." International Journal of Heat and Mass Transfer 136 (2019): 298311. https://doi.org/10.1016/i.ijheatmasstransfer.2019.02.105

[44] Q. Song, G. Chen, H. Guo, J. Shen, and M. Gong. "Two-phase flow condensation pressure drop of R14 in a horizontal tube: Experimental investigation and correlation development." International Journal of Heat and Mass Transfer 139 (2019): 330-342. https://doi.org/10.1016/j.ijheatmasstransfer.2019.05.023

[45] M. C. Leverett. "Capillary Behavior in Porous Solids." SPE-941152-G 142, no. 1 (1941): 341-358. https://doi.org/10.2118/941152-G

[46] Shekarriz, A., and O. A. Plumb. "A theoretical study of the enhancement of filmwise condensation using porous fins." In AIAA-ASME 4th Joint Conference, Boston, June, pp. 1-4. 1986.

[47] C. Y. Wang and C. Beckermann. "Boundary Layer Analysis of Buoyancy-Driven Two-Phase Flow in Capillary Porous Media." Journal of Heat Transfer 117, no. 4 (1995): 1082-1087. https://doi.org/10.1115/1.2836290

[48] R. H. Brooks and A. T. Corey. "Properties of porous media affecting fluid flow." Journal of the Irrigation and Drainage Division 92, no. 2 (1866): 61-90.

[49] Plumb, O. A. "Heat transfer during unsaturated flow in porous media." In Convective heat and mass transfer in porous media, pp. 435-464. Springer, Dordrecht, 1991. https://doi.org/10.1007/978-94-011-3220-6 13 